\section{Life at the scrutiny of optical forces}

Pierre-François Lenne and Pierre Mangeol

Aix Marseille Univ, CNRS, IBDM, Marseille France, Turing Center for Living Systems, Marseille France

Corresponding author: pierre-francois.lenne@univ-amu.fr

\section{Abstract}

In this short review we give the historical Optical tweezers have been celebrated in 2018 background of optical tweezers, examine their with the Nobel Prize in Physics awarded to Arthur physical principles and describe in a few examples Ashkin. We trace here the history of this technique, how optical tweezers uncovered the mechanical which has proven essential for the discovery of world that takes place in our cells.

several mechanisms of Life. The technique relies

on optical forces and results from the momentum transfer between light and objects, usually of micrometer size. Particularly suitable for the study of molecular machines, optical tweezers also shed light on the mechanics of cells in vitro or in living organisms.

\section{Introduction}

At the cellular scale, Life is made of molecular nuts and bolts, beams, tiny machines that build, degrade, transport and organise. Light and electron microscopy are the methods of choice to observe the cell, but they keep us from a distance to this mechanical world. Directly probing the mechanics of the cell and of its components is therefore crucia to understand the basic Life processes.

In the last 30 years a few techniques arose in biophysics labs to tackle this important problem. Among them, optical tweezers became more an more popular because of their high versatility. They can probe mechanics from the cellular leve down to a single molecule, with a high tempora and spatial resolution. Because they are based on light, they are hardly intrusive for the sample. With an increasing number of companies providing plugand-play solutions, optical tweezers are becomin accessible to a wider community.

\section{History}

Optical tweezers are a relatively new technique, but their historical grounds can be traced back to the early $17^{\text {th }}$ century. In 1607 Johannes Kepler watched Halley's comet with fascination and two years later hypothesized that the comet tail points away from the sun because it is pushed by the solar radiation (Figure I). This profound observation led him to push to travel: "Provide ships or sails adapted to the heavenly breezes, and there will be some who will brave even that void". However, the theoretical formulation of this "push" and its experimenta confirmation had to wait almost three centuries. In 1873 Maxwell formulated that light as a wave can exert a force on a massive object by transfer of momentum. In a back-of-the-envelope calculation, Maxwell estimated that the sunlight presses earth's surface with a force of around four pounds per square mile, that is equivalent to $0.7 \mathrm{~kg} / \mathrm{km}^{2}$. In 1901 , Lebedev provided a direct proof of this theoretical discovery, realising an experimental tour-de-force with tiny mirrors and optical elements (Lebedew 1901).The pressure measured with the light sources of the time was, however, rather small, leading Poynting to say, in his presidential address to British Physical Society in 1905, that the minuteness of optical forces puts them "beyond consideration in write to Galileo that humans may, one day, use this

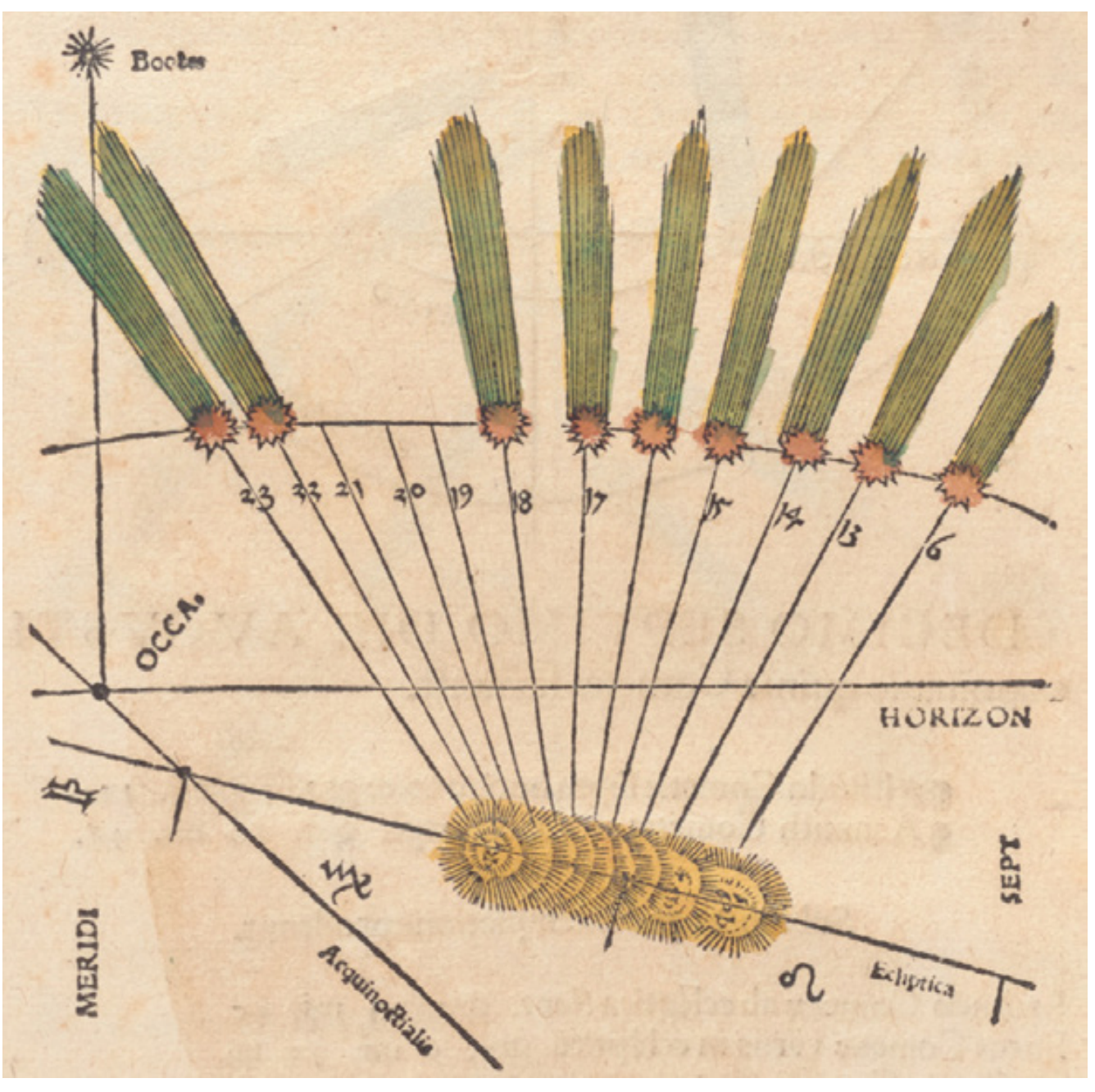

Figure I. Drawing from Peter Apian in his book Astronomicum Casareum (I540) showing that a comet's tail points away from the Sun.Th comet was observed in I53I and is now known as Halley's Comet.

terrestrial affairs". This was without counting on the invention of lasers in 1960s, which revolutionized optics by providing light sources with high spatial coherence and thus high "focusability". In 1970, Ashkin reported a seminal experiment that demonstrated the ability of light to accelerate and trap micrometer-size particles (Ashkin 1970). The experimental set-up consisted of a laser of 19 $\mathrm{mW}$ focused by an objective lens into a chamber containing polystyrene beads dispersed in water (Figure 2). Ashkin observed beads pushed and accelerated in the direction of the beam propagation, but also beads transiently attracted by the beam towards the high intensity region. In this scheme, the trapping force was insufficient to stabilise the beads against radiation pressure, and Ashkin used two counterpropagating beams to produce a stable aser trap. Optical tweezers were born. The singlelens optical trap, which is nowadays commonly used for confinement, was invented in 1986. The crucial element, which was absent from the 1970 Ashkin's experiment, is a high numerical objective lens that allows a tight focusing of the beam onto particles. With such traps, dielectric particles in the size ranging from $10 \mu \mathrm{m}$ down to $25 \mathrm{~nm}$ were stably trapped in water solution. This opened the way to trap, in three dimensions, particles of very different sizes, from macromolecules to biological cells. This was also instrumental to manipulate and cool down atoms. 


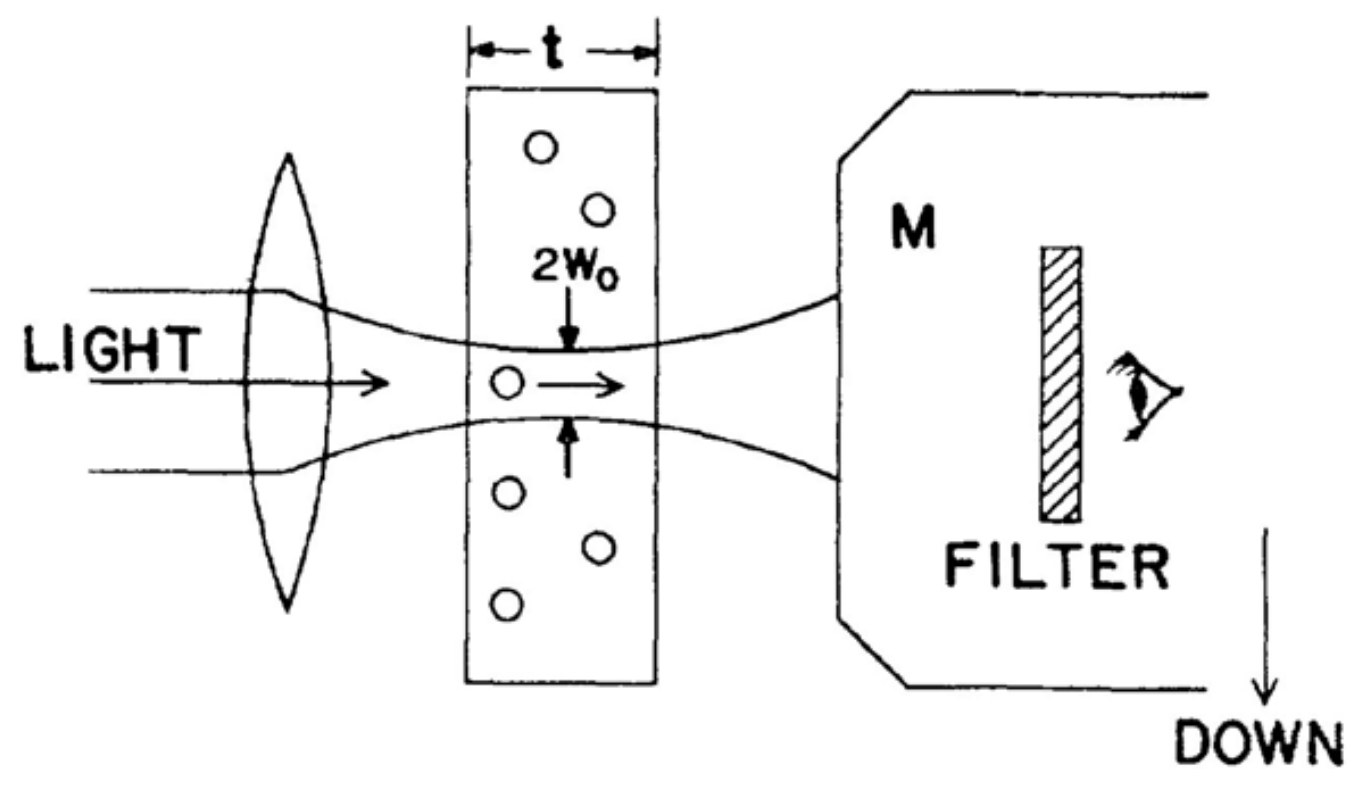

Figure 2. Ashkin's schematic of his seminal experiment on manipula
and pushes them in the direction of light propagation (Ashkin, 1970)

\section{Principles}

In 1970, Ashkin identified two basic forces produced by light on the particles: a scattering force that pushes particles in the direction of the incident beam and a gradient force along the intensity gradient that pulls particles back towards the brightest spot of the beam. The scattering force is due to the transfer of momentum of the light to the particle as a result of scattering and absorption processes. The gradient force is responsible for confinement. For dielectric particles, the gradient force arises from the fact that dipoles in a non-uniform beam experience forces in the direction of the field gradient. For particles whose size are much larger than the wavelength of the trapping laser (Mie scattering), the ray optic picture illustrates the origin of the gradient force produced by the non-uniform trapping beam. Refraction of the incident light by the spherica particle leads to a change of momentum carried by the beam; in turn, by momentum conservation, an opposite momentum is imparted to the bead (Figure 3). For particles whose refractive index is higher than the surrounding medium, particles are attracted towards the high intensity region of the beam. For particles whose refractive index is lowe made possible by focusing the trapping laser to diffraction-limited spot using an objective lens of high numerical aperture. For small displacements $(<200 \mathrm{~nm})$ the restoring force due to the intensity gradient is proportional to the offset from the equilibrium position. As a consequence, the optical trap acts as a Hookean spring whose stiffness is proportional to the light intensity

Trapping forces on the order of I pN per $10 \mathrm{~mW}$ of power using a I.2 NA objective lens are achieved with micrometer size beads. The typical trap stiffness is on the order of $100 \mathrm{pN} / \mu \mathrm{m}$.

The development of optical tweezers is strongly linked to the development of new technologies, specifically lasers, objective lenses, piezoelectric stages and position detectors. Building an optical weezers setup is quite straightforward, yet quantitative measurements with high accuracy requires expertise in optics and data acquisition (Neuman and Block 2004).

\section{Applications}

Applications of optical trapping are numerous and their scientific impact has been important in several fields. Ashkin was awarded the Physics Nobel prize

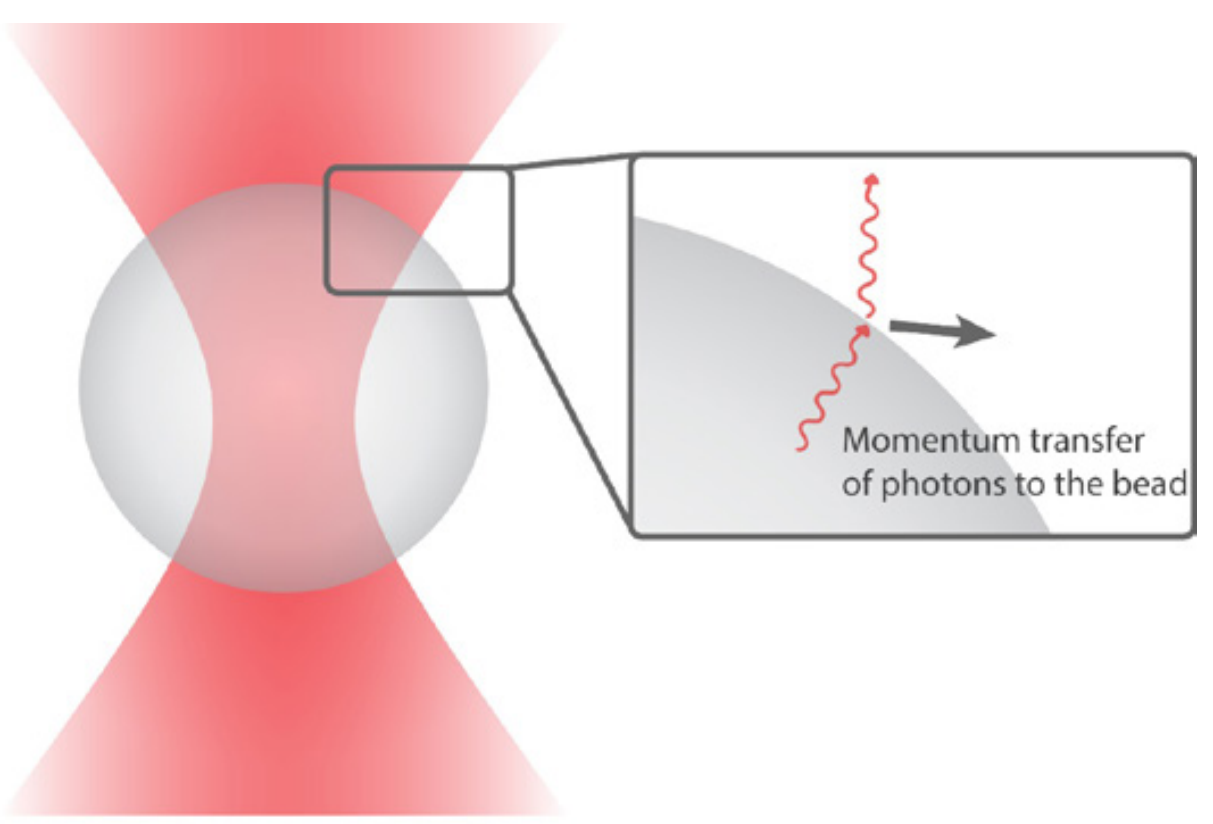

2018 for his discovery of optical tweezers and their applications in biological systems, while two areas of physics that directly benefited from his discovery were previously awarded this prize: laser cooling and trapping of atoms (1997), and the realisation of Bose-Einstein condensates (2001).We will focus on the applications of optical tweezers in life sciences, but we shall not forget their major ontribution to physics, nanotechnology and soft

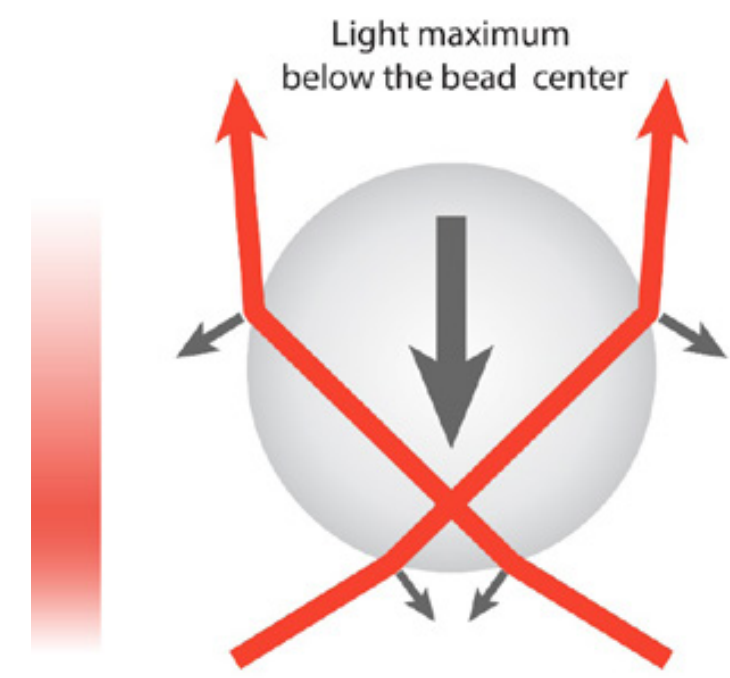

Light gradient toward right

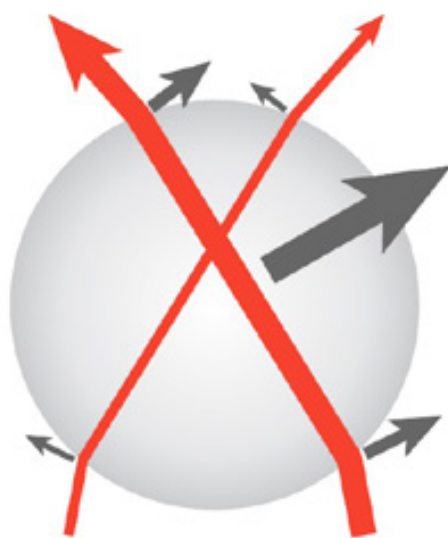

Figure 3. Principles: Optical trapping lies in the ability of light to transfer its momentum to matter.A particle is attracted towards the maximu of intensity of the trapping beam. 
a

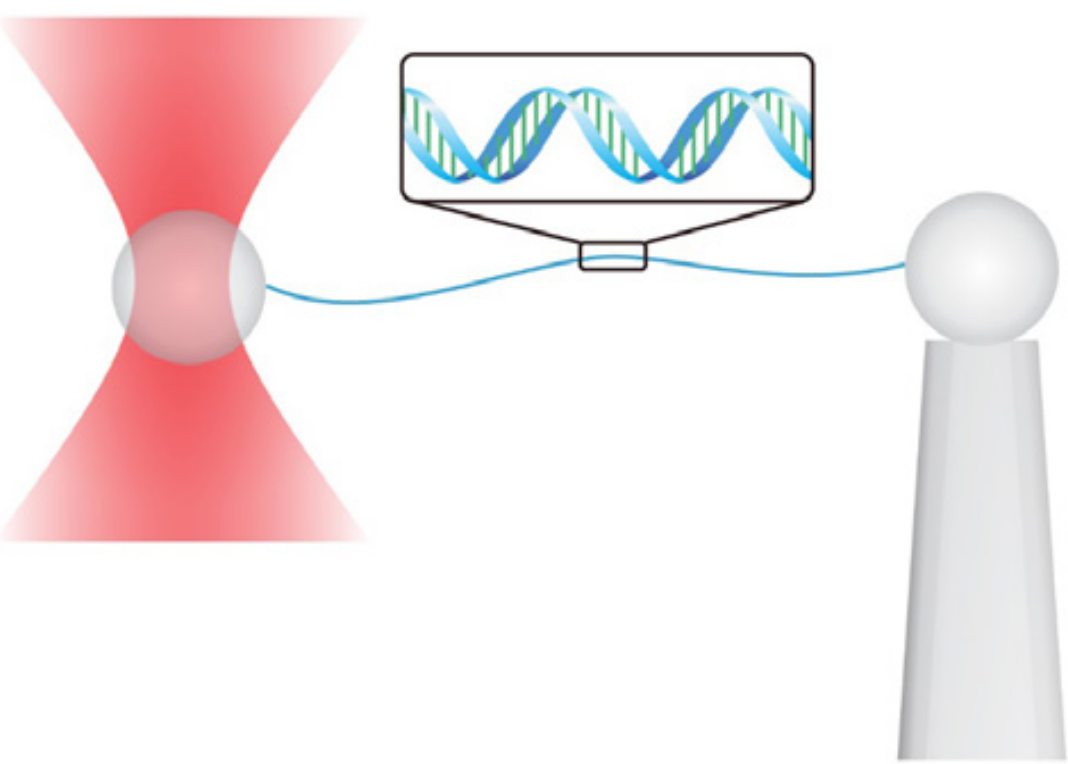

b

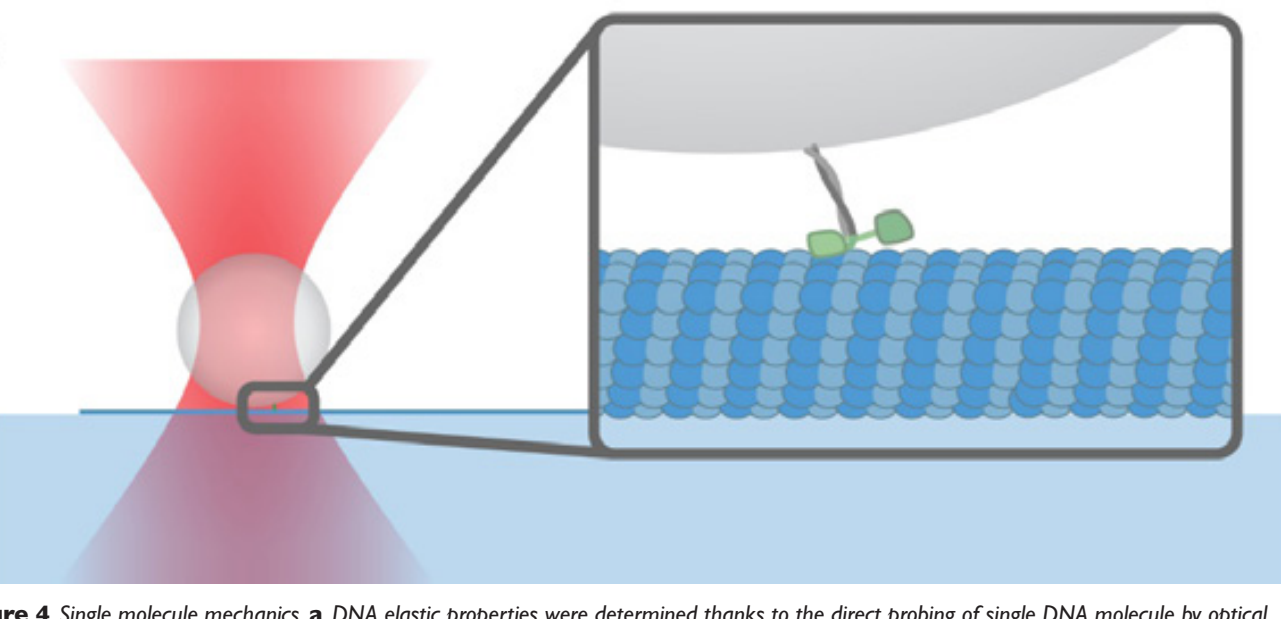

Figure 4. Single molecule mechanics. a, DNA elastic properties were determined thanks to the direct probing of single DNA molecule by optica tweezers. b. When attached to a bead, kinesins or myosins exert a pulling force detected by optical tweezers, giving access to the stepping mechanism of these molecular motors.

matter (See Jones and others (2015), a recently happened to be contaminated with bacteria. To published book which covers most applications, as his and his team's greatest surprise they managed well as the principles of the technique).

The application of optical tweezers to biology did not occur immediately because of the belief of many that the intense laser light needed for trapping would not be compatible with life. Therefore the first application of optical tweezers to biology happened against all odds and actually with a bit of luck. As Arthur Ashkin recalled in a conference he gave to the Optical Society of America in 2014, a sample he left overnight to use the next mornin to capture bacteria with optical tweezers without damaging them (Ashkin and Dziedzic 1987). This seminal observation led to numerous application that have flourished since then, including the application of optical tweezers to single-molecule biophysics, cell biology and medicine.

\section{Single molecule biophysics}

For many years, the studies of molecules found in the cell were done in bulk. Researchers would a
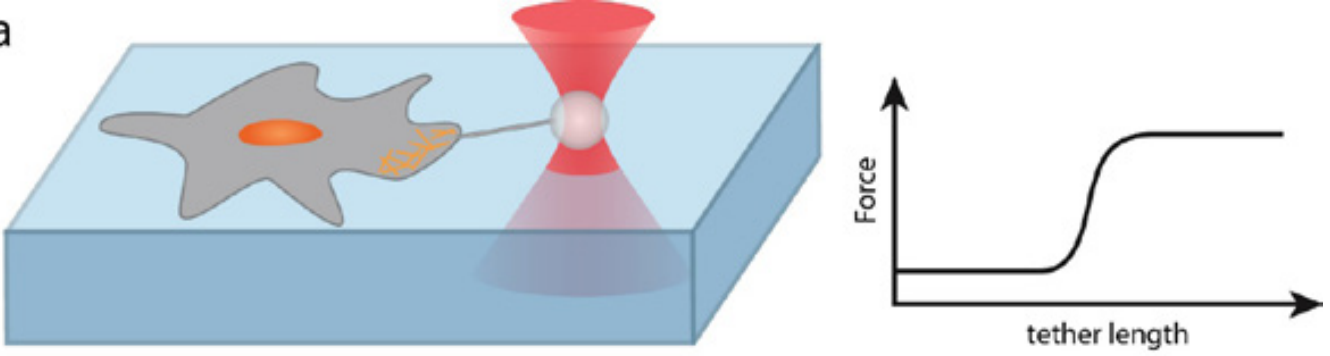

b

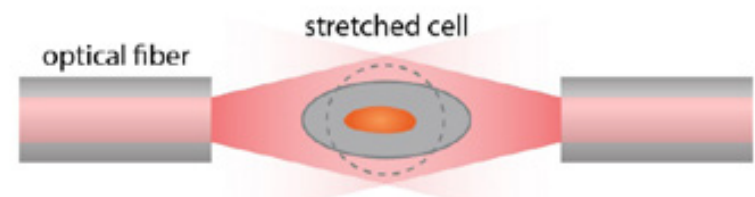

C
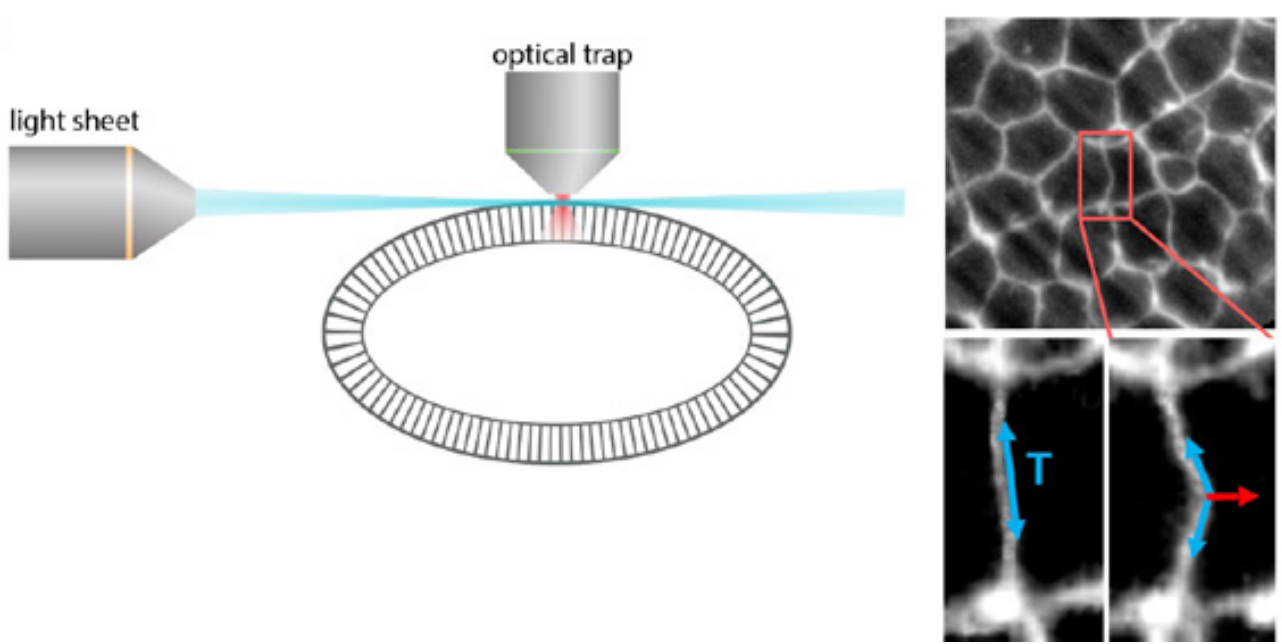

Figure 5. Probing cell mechanics. a, A membrane tether can be pulled out from the cell surface.As the tether initiates, the force applied on the bead increases suddenly; when the tether lengthens, the force stays constant, indicating a mechanical transition. b. The dual fiber apparatus allows a cell to be stretched, which is attracted by the light source emanating from both optical fibers. $\boldsymbol{c}$, Cell junctions can be trapped directly. Here the and rheological properties The combination of a light-sheet scheme with an optical trap allows for the high signalto-noise ratio in vivo imaging as the cell mechanics is probed.

only have access to the average behaviour of a very large number of molecules at the same time. In the 70 s and 80 s, together with optical tweezers, a few techniques flourished to access to the behaviour of single molecule at a time. Patch clamp technique could probe single ion channels activity, optical tweezers, magnetic tweezers and atomic force microscopy could mechanically probe single proteins, polymers or nucleic acids, while a number of highly sensitive fluorescence techniques allowed

the detection of single molecules.

The success of optical tweezers in this area lies in the length (from a fraction of $n m$ to a few tens of micrometers) and force scales (from a fraction to $\mathrm{pN}$ to a few hundreds of $\mathrm{pN}$ ) at which optical tweezers operate and match those at play in the molecular world, whose natural units are $\mathrm{nm}$ and $\mathrm{pN}$. The natural energy unit for single molecules (DNA, molecular motors) is the thermal energy $\mathrm{kT}$, where $\mathrm{k}$ is the Boltzmann constant and $\mathrm{T}$ the 
temperature. Any motion per degree of freedom that a molecule can make is stimulated by one hal of this energy. At room temperature, $\mathrm{kT}=4 \mathrm{10}{ }^{\wedge}$ 2I J which can be conveniently expressed as the mechanical work of a force (in $\mathrm{pN}$ ) by a distance (in $n m): k T=4$ pN.nm. Optical tweezers currently used in labs can typically probe energies that are a tenth of the thermal energy.

\section{DNA}

Single molecule experiments using optical tweezers use beads as handles, with single molecules attache to their surface through chemical bonds. Fo example, to probe the elasticity of DNA molecules, Smith et al. (1996) held a single molecule between two beads: one immobilised by a micropipette, the other trapped by optical tweezers (Figure 4a). The molecule is stretched by moving the micropipette, while the force is determined by measurin the displacement of the trapped bead from the trap centre. Force-extension curve reveals the mechanical properties of the probed molecules, such as the elastic modulus and the persistence length, which characterises the length of bending fluctuations.

\section{Molecular motors}

Another impressive achievement of optical tweezers is the understanding of the mechanics of single molecular motors. These proteins are widely found in the cell and carry out major functions, such as DNA replication by DNA polymerases, muscle contraction by myosins or long-range transport by kinesins. Many different schemes have been used to probe these proteins with optical tweezers The most typical experiment is to chemically attach a very few motors on a single bead and then approach the bead to the substrate of choice of the motor (Figure 4b). Grabbing the protein by its tail, number of labs could observe that the bead is pulled by the motor unidirectionally along its substrate. They could follow stepping mechanisms of kinesin (Block and others 1990; Svoboda and others 1993) or myosins (Mehta and others 1999) in real time and observe complex behaviours that were not even imagined from results of bulk experiments. Proteins can pause on their substrate or go backward (such as in the case of DNA polymerases that step back on the DNA to check for errors (Wuite and others 2000; Hoekstra and others 2017). In early studies, researchers even found that in a population of seemingly identical motor proteins, some motors perform better than others (Wuite and others 2000).

\section{Cell biology and development}

Optical tweezers have proven to be a powerful and versatile tool to probe cell mechanical properties and cell interactions. One example is the study of adhesion between cells and their environment Adhesion plays a crucial role in how cells organise in tissues and organs, and how cells migrate in the organism. Cells adhere to the extracellular matrix through molecules called integrins which link the intracellular skeleton of cells (cytoskeleton) to proteins from the extracellular matrix. To probe the strength of interactions between integrin and the cytoskeleton, trapped beads were coated with an integrin domain and brought into contact with the leading edge of a migrating cell (Jiang and others 2003). Upon migration the bead is displaced from the trap centre as a result of the force acting between integrin and the cytoskeleton. When a link is broken, the bead is pulled back to the trap centre. Force measurements during these events are informative on the strength of the link (typically of the order of $2 \mathrm{pN}$ ).

Using beads attached to cell membranes, it is also possible to measure the mechanical properties of cell membranes. Interestingly, pulling a bead attached to a membrane away from the cell leads to the formation of narrow tubes, called membrane tethers (Dai and Sheetz 1995) (Figure 5a).The force at which the tether forms is indicative of the interaction between the membrane and the cytoskeleton The tethers can be dramatically elongated with a constant force (membrane tension) that indicates the existence of a membrane reservoir.
Single cells and organelles can be directly trapped as their constituents offer sufficient refraction index mismatch from the surrounding.

Interestingly, focused lasers are not always required for efficient for trapping. One particularly efficient scheme to trap objects consists in two optical fibres which deliver two divergent counter-propagating beams (Constable and others 1993). Using this device, cells or cell clusters as large as $100 \mu \mathrm{m}$ in diameter can be trapped and stretched (Guck and others 200I) (Figure 5b)

More recently optical tweezers have been applied to measure cell mechanics in vivo during the development of an organism, namely the fruit fly Drosophila melanogaster (Bambardekar and others 2015). Combined with light sheet microscopy the approach provides measures of tension and material properties of cell-to-cell contacts over the course of animal development (Figure 5c). An important finding is that cell-to-cell contacts behave like elastic solids at timescales of less than a few tens of seconds and viscous liquids at longer times (Clément and others 2017). They are therefore able to withstand short-term forces but can be irreversibly remodelled under the application of long-term forces.

\section{Concluding remarks}

Laser tweezers have a long history but live a Renaissance as illustrated by recent biological applications - but also optical developments including holographic approaches or combination with fluorescence imaging that we have not covered in this review. "May the optical forces be with you!", or at least, may the optical tweezers be in the toolkit of life scientists.

\section{References}

Ashkin, A. 1970. 'Acceleration and Trapping of Particles by Radiation Pressure', Physical Review Letters, 24.4: 156-59 <http://dx.doi.org//0.1103/ PhysRevLett.24.156>

Ashkin, A, and J M Dziedzic. 1987. 'Optical Trapping and Manipulation of Viruses and Bacteria., Science (New York, N.Y.), 235.4795: 15।7-20

Bambardekar, Kapil, Raphaël Clément, Olivier Blanc, Claire Chardès, and Pierre-François Lenne. 2015. 'Direct Laser Manipulation Reveals the Mechanics of Cell Contacts in Vivo.', Proceedings of the National Academy of Sciences of the United States of America <http://dx.doi.org// 0.1073/pnas.1418732/ I2>

Block, S M, L S Goldstein, and B J Schnapp. 1990. 'Bead Movement by Single Kinesin Molecules Studied with Optical Tweezers.', Nature, 348.6299 (Nature Publishing Group): 348-52 <http://dx.doi. org//0.1038/348348a0>

Clément, Raphaël, Benoît Dehapiot, Claudio Collinet, Thomas Lecuit, and Pierre-François enne. 2017. 'Viscoelastic Dissipation Stabilizes Cell Shape Changes During Tissue Morphogenesis.', Current Biology: CB, 27.20: 3132-34 <http://dx.doi. org//0.1016/j.cub.2017.09.005>

Constable, A, J Kim, J Mervis, F Zarinetchi, and M Prentiss. 1993. 'Demonstration of a Fiber-Optical Light-Force Trap., Opt Lett, I8.2 I: 1867-69

Dai, J, and M P Sheetz. 1995. 'Mechanical Properties of Neuronal Growth Cone Membranes Studied by Tether Formation with Laser Optical Tweezers.' Bioph. J, 68.3: 988-96 <http://dx.doi.org/10.1016/ S0006-3495(95)80274-2>

Guck, J, R Ananthakrishnan, H Mahmood,T J Moon, C C Cunningham, and others. 200I. 'The Optical Stretcher: a Novel Laser Tool to Micromanipulate Cells.', Bioph. J, 81.2: 767-84 <http://dx.doi. org/10.1016/50006-3495(0I)75740-2>

Hoekstra, Tjalle P, Martin Depken, Szu-Ning Lin, ordi Cabanas-Danés, Peter Gross, and others. 2017. 'Switching Between Exonucleolysis and Replication by T7 DNA Polymerase Ensures High Fidelity.', Biophysical Journal, II2.4: 575-83 <http://dx.doi. org/10.1016/j.bpj.2016.12.044>

Jiang, Guoying, Grégory Giannone, David R Critchley, Emiko Fukumoto, and Michael P Sheetz. 2003. 'Two- 
Piconewton Slip Bond Between Fibronectin and the Cytoskeleton Depends on Talin., Nature, 424.6946 (Nature Publishing Group): 334-37 <http://dx.doi. org/| 0.1038/nature0|805>

Jones, Philip, Onofrio Maragó, and Giovanni Volpe. 2015. Optical Tweezers (Cambridge University Press)

Lebedew, Peter. 190I. 'Untersuchungen Über Die Druckkräfte Des Lichtes', Annalen Der Physik, 3II.II (Wiley-Blackwell): 433-58 <http://dx.doi. org/|0.1002/andp. 190|3||||102>

Mehta, A D, R S Rock, M Rief, J A Spudich, M $S$ Mooseker, and others. 1999. 'Myosin-v Is a Processive Actin-Based Motor.', Nature, 400.6744 (Nature Publishing Group): 590-93 <http://dx.doi. org/10.1038/23072>

Neuman, Keir C, and Steven M Block. 2004. 'Optical Trapping', Rev Sci Instrum, 75.9: 2787-2809 <http://

\section{Submit to infocus}

infocus welcomes submissions of articles of general interest to microscopists.

You provide the text and images and we take care of the rest. It's the ideal way to share your work with the microscopical community.

Full submission information and guidelines are available at

www.infocus.org.uk. To submit an idea or if you have any questions about the process please email the Editor (editor@infocus.org.uk)
Smith, S B, Y Cui, and C Bustamante. 1996. 'Overstretching B-DNA: the Elastic Response of Individual Double-Stranded and Single-Stranded DNA Molecules.', Science (New York, N.Y.), 271.5250

Sroboda, K, C F Schmidt, B J Schnapp, and S M Block. Interferometry.', Nature, 365.6448 Wuite, G J, S B Smith, M Young, D Keller, and C Bustamante. 2000. 'Single-Molecule Studies of the Effect of Template Tension on T7 DNA Polymerase org/| 0.1038/350036|4>
Labtech-EM... ...innovative and practical tools for electron microscopists

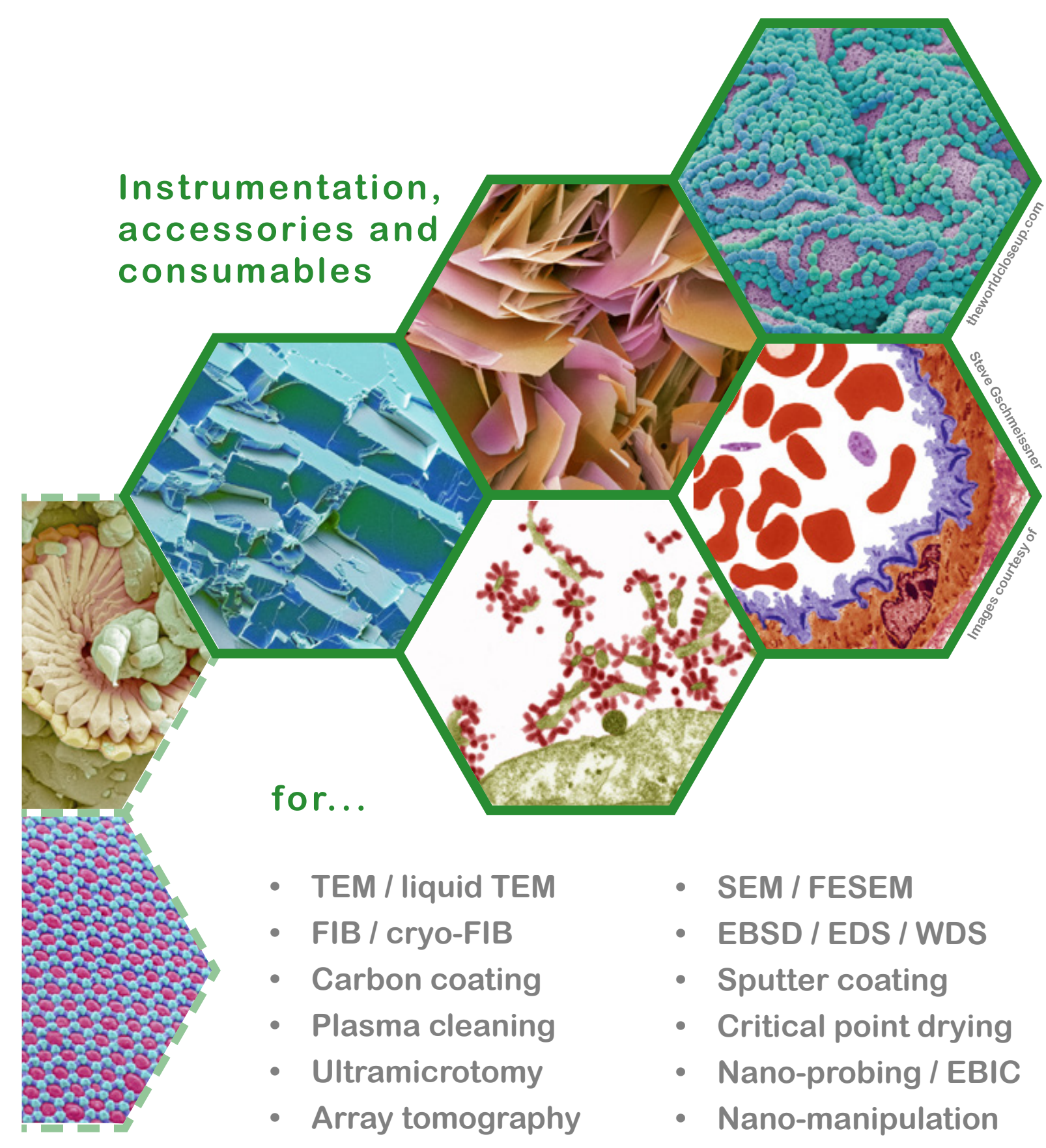

